\title{
Effects of Holdups of Gas and Liquid Phases in Packed-bed Electrode on Current Efficiency of Electrochemical Production of Hydrogen Peroxide
}

\author{
Tsutomu HIBINO and Masao SUDOH*
}

Received May 30, 1997 ; Accepted September 2, 1997

\begin{abstract}
A packed-bed electrode of graphite felts sparged with oxygen gas was used for the on-site electrochemical production of hydrogen peroxide. The terminal voltage and the current efficiency were affected by operational conditions such as liquid and gas velocities in the packed-bed cathode. The holdups of gas and liquid phases, and the potential profiles of the solution phase in the cathode were measured to clarify the effects of the operational conditions on the electrolysis performance. The holdups of gas and liquid phases were determined as functions of superficial velocities of gas and liquid. The cathodic potential in the bed was shifted up by increasing liquid velocity at a certain velocity of gas. The reaction resistivity of oxygen reduction was increased by increasing liquid holdup, because of the increase in the diffusion length of the oxygen. Meanwhile the effective resistivity of the solution was decreased by increasing liquid holdup. Then the potential of the solution in the bed had a minimum profile at a certain holdup of the liquid phase. The optimum conditions for electrolysis were determined as follows: the current density was $300 \mathrm{~A} \cdot \mathrm{m}^{-2}$, holdups of gas and liquid phases, $\varepsilon_{G}=0.85 \sim 0.90$, and $\varepsilon_{L}=0.04 \sim 0.05$.
\end{abstract}

\section{INTRODUCTION}

Hydrogen peroxide had been produced by electroreduction of oxygen in an alkaline solution with the trickle-bed electrode ${ }^{1)}$, the packed-bed electrode $\mathrm{e}^{2-5}$ ) or the gas-diffusion electrode ${ }^{6,7)}$. The polarization characteristics of the packed-bed reactor without gassparging $^{2)}$, the production of acidic hydrogen peroxide by using a 3 -compartment cell ${ }^{4,5}$ ) and the comparison among the packing materials for the oxygen-sparged cathode ${ }^{3)}$ were reported in our previous papers. Cathodic reduction of oxygen in alkaline solutions on the graphite electrode proceeds by successive and competitive reactions as follows:

$$
\begin{gathered}
\mathrm{O}_{2}+\mathrm{H}_{2} \mathrm{O}+2 \mathrm{e}^{-} \rightleftharpoons \mathrm{HO}_{2}^{-}+\mathrm{OH}^{-} \\
\mathrm{HO}_{2}^{-}+\mathrm{H}_{2} \mathrm{O}+2 \mathrm{e}^{-} \rightleftharpoons 3 \mathrm{OH}^{-}
\end{gathered}
$$

${ }^{*}$ Department of Materials Science and Chemical Engineering, Shizuoka University (Hamamatsu 432, Japan)

Key Words: Electrolysis, Hydrogen peroxide, Packedbed electrode, Holdup
Since the peroxide ion is an intermediate substance for the reduction of oxygen, it is possible for peroxide ions to accumulate to higher concentration when reaction (1) is faster than reaction (2). But when cathodic overpotential increase, reaction rate of (2) is increased, then current efficiency is decreased by decomposition of hydrogen peroxide. ${ }^{2)}$

Graphite felts had some advantages of low cost and easy maintenance for the electrode exchange, but the lower current density compared to the gas-diffusion electrode. Hydrogen peroxide produced by an on-site facility is useful to the pulp bleaching ${ }^{1)}$, an advanced wastewater treatment ${ }^{8,9)}$ and so on. The relationship between the current efficiency of production of hydrogen peroxide and flow properties of the gas-liquid phase in the packed-bed has not been clarified in detail so far.

The purpose of this paper is to measure the holdups of gas and liquid phases, and the 


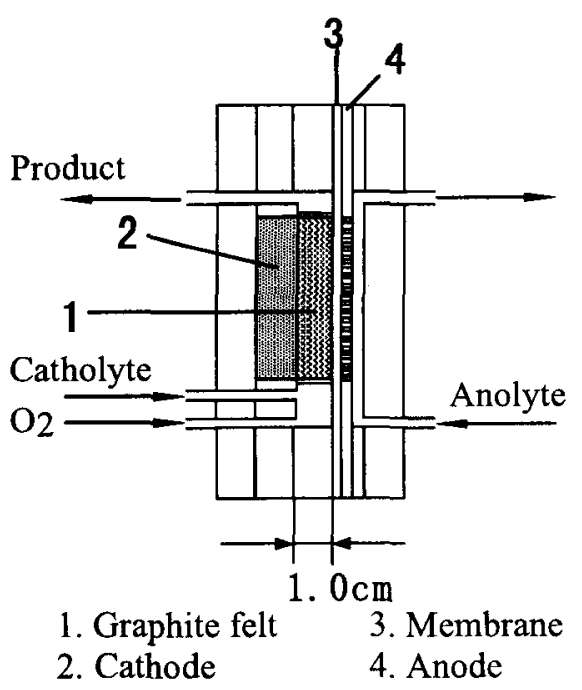

Fig.1 Packed-bed electrode.

potential profiles of the solution phase in the oxygen-sparged cathode, and to clarify the effects of the operational condition on the electrolysis performance.

\section{EXPERIMENTAL}

\subsection{Packed-bed reactor}

The details of packed-bed electrode reactor are shown in Fig.1. The cathode was a packed-bed electrode of graphite felts (GF-20/5F, Nippon Carbon) and a graphite feeder, $5 \mathrm{~cm}$ wide, $10 \mathrm{~cm}$ high. The bed depth, the distance from the feeder to the separator, was $1 \mathrm{~cm}$. A nickel plate was used as the anode. The cathode compartment was separated from the anode compartment by a cation-exchange membrane (Nafion 117, Dupont).

Figure 2 shows a schematic diagram of experimental apparatus. The catholyte was $0.1 \mathrm{M} \mathrm{NaOH}$ and the anolyte was $1.0 \mathrm{M}$ $\mathrm{NaOH}$ solution. Saturated oxygen gas was sparged from the bottom of the cathode compartment. The catholyte was one pass

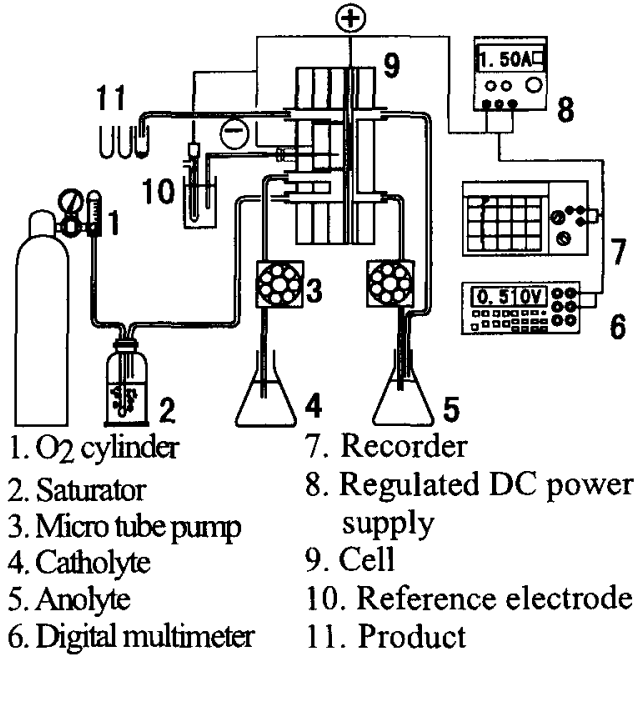

Fig.2 Schematic diagram of experimental apparatus.

flow through the bed, the anolyte was recycled between the tank and the reactor for a certain period before exchange. Electrolysis was carried out at a room temperature by the constant current method, and the terminal voltage was monitored during electrolysis. The concentration of hydrogen peroxide was determined by measuring the light absorption of the titanic-hydrogen peroxide colored complex at $410 \mathrm{~nm}$ with a spectrophotometer. ${ }^{2)}$ The cathodic potential distribution in the bed was measured by a Luggin capillary connected to the reference electrode, an $\mathrm{Ag} / \mathrm{AgCl}$ electrode in a saturated $\mathrm{KCl}$ solution, inserted from the back of the feeder electrode.

\subsection{Electrolysis}

The relationship between the current density, based on the surface area of the feeder electrode, and the terminal voltage was obtained under the following condition: the superficial velocities of the catholyte and the anolyte, $u_{L c}$ and $u_{L a}$, were $0.002 \mathrm{~cm} \cdot \mathrm{s}^{-1}$ and $0.03 \mathrm{~cm} \cdot \mathrm{s}^{-1}$ respectively, and the superficial 
velocity of gas $u_{G}$ was $1.7,5.0,6.7$ and 10 $\mathrm{cm} \cdot \mathrm{s}^{-1}$. The current efficiency of hydrogen peroxide production was obtained by onehour electrolysis at various current densities, $300,400,600,800$ and $1000 \mathrm{~A} \cdot \mathrm{m}^{-2}$, and $u_{L c}$, $u_{L a}$ and $u_{G}$ were $0.002,0.03$ and $5.0 \mathrm{~cm} \cdot \mathrm{s}^{-1}$ respectively.

\subsection{Holdups of gas and liquid phases}

Holdups of gas and liquid phases were measured under the constant superficial velocity of anolyte and catholyte; $u_{L a}=0.03 \mathrm{~cm} \cdot \mathrm{s}^{-1}$, $u_{L c}=0.002 \mathrm{~cm} \cdot \mathrm{s}^{-1}$, and different superficial velocity of gas $u_{G}, 1.67,5.00,8.33$, 13.3 and $20.0 \mathrm{~cm} \cdot \mathrm{s}^{-1}$. The concentration of the hydrogen peroxide produced, the terminal voltage, and the potential of the solution phase were measured at different conditions; $u_{L a}=0.03 \mathrm{~cm} \cdot \mathrm{s}^{-1}, u_{G}=5.00 \mathrm{~cm} \cdot \mathrm{s}^{-1}$, and $u_{L c}=0.0020,0.0057,0.0097,0.0140,0.0183$, $0.0389,0.0553$ and $0.0833 \mathrm{~cm} \cdot \mathrm{s}^{-1}$.

Holdups of gas and liquid phases, $\varepsilon_{G}$ and $\varepsilon_{L}$, were estimated as follows. At first only dry oxygen was flowed into the cathode compartment. Then the dry oxygen was switched to the wet oxygen, as soon as the catholyte was flowed into the cathode compartment. The period $t$, from this change to the time when the catholyte flooded from the outlet, was measured. The holdup of the liquid phase $\varepsilon_{L}$ was estimated with the superficial velocity of the catholyte $u_{L c}\left[\mathrm{~cm} \cdot \mathrm{s}^{-1}\right]$, the cross section of packed-bed $S\left[\mathrm{~cm}^{2}\right]$, the volume of packed-bed $V\left[\mathrm{~cm}^{3}\right]$, and the flooding time $t[\mathrm{~s}]$.

$$
\varepsilon_{L}=u_{L e} S t / V
$$

Then the holdup of gas phase $\varepsilon_{G}$ was estimated as follows:

$$
\varepsilon_{G}=1-\varepsilon_{S}-\varepsilon_{L}
$$

The value of $\varepsilon_{S}$, the holdup of solid phase of the electrode, was given as $0.076^{3)}$ in this work.

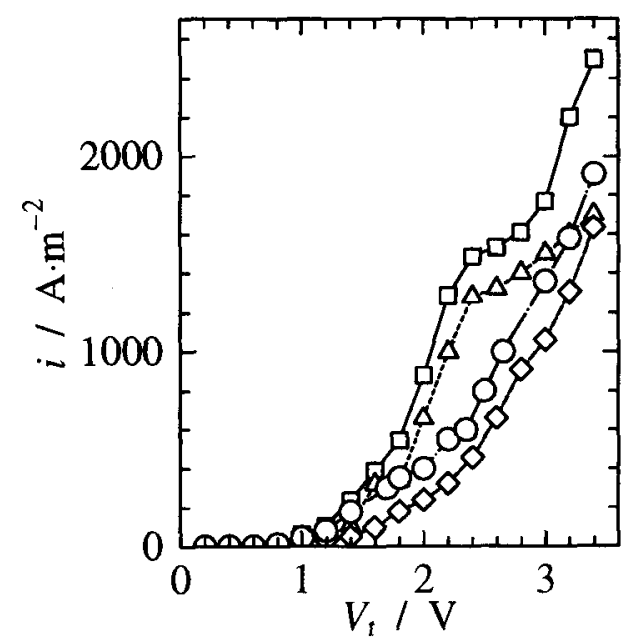

Fig.3 Relationship between current density and terminal voltage. The superficial velocity of gas $u_{G}$ was $(\square) 10,(\triangle) 6.7,(\bigcirc) 5.0$, and $(\diamond) 1.7 \mathrm{~cm} \cdot \mathrm{s}^{-1}$. The superficial velocities of catholyte and anolyte, $u_{L c}$ and $u_{L a}$, were 0.002 and $0.03 \mathrm{~cm} \cdot \mathrm{s}^{-1}$ respectively.
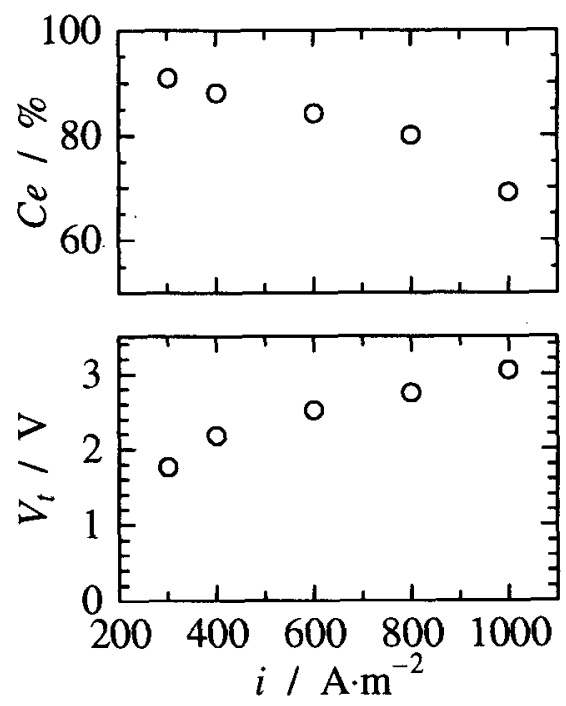

Fig.4 Effects of current density on current efficiency and terminal voltage. The superficial velocity of gas $u_{G}$ was $5.00 \mathrm{~cm} \cdot \mathrm{s}^{-1}$, and electrolyte was the same as in Fig. 3 . 

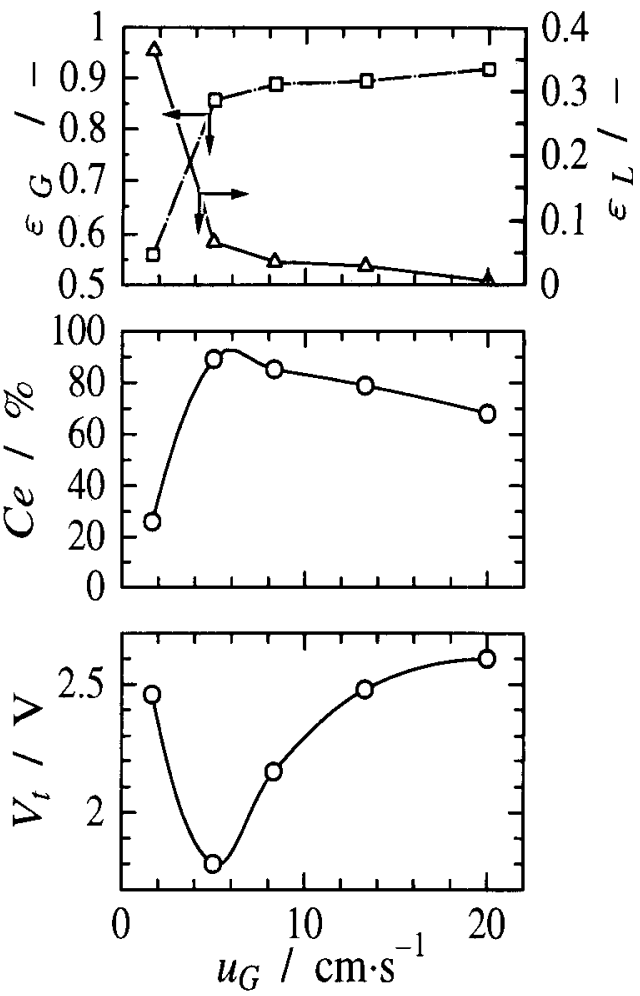

Fig.5 Effects of superficial velocity of gas on holdups, current efficiency and terminal voltage. The superficial velocities of catholyte and anolyte, $u_{L c}$ and $u_{L a}$, were 0.002 and 0.03 $\mathrm{cm} \cdot \mathrm{s}^{-1}$ respectively. The current density $i$ was $300 \mathrm{~A} \cdot \mathrm{m}^{-2}$.

\section{RESULTS AND DISCUSSION}

\subsection{Effect of current density on cur- rent efficiency}

Figure 3 shows the relationship between the current density and the terminal voltage at different values of $u_{G}$. The current density was increased with increasing $u_{G}$. Since the concentration of the dissolved oxygen on the electrode surface was increased by increasing $u_{G}$, the production rate of hydrogen peroxide and the current density were increased.

Figure 4 shows the effect of the current density on the current efficiency and the terminal voltage. The current efficiency $\mathrm{Ce}[\%$ ] was calculated according to the following

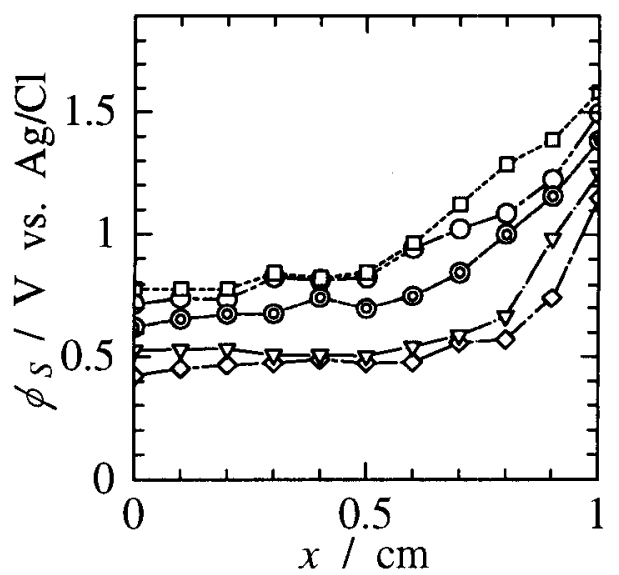

Fig.6 Cathodic potential profiles of solution phase in packed-bed electrode. The superficial velocity of gas $u_{G}$ was $(\square) 20.0$, ( $\left.\bigcirc\right) 1.67$, (O) $13.3,(\nabla) 8.33$, and $(\diamond) 5.00 \mathrm{~cm} \cdot \mathrm{s}^{-1}$. Experimental condition was the same as in Fig. 5.

equation:

$$
C e=2 F Q C_{p} / I \times 100
$$

where $F$ Faraday's constant $\left[\mathrm{C} \cdot \mathrm{mol}^{-1}\right], Q$ the volumetric flow rate of the catholyte $\left[\mathrm{m}^{3}\right.$. $\left.\mathrm{s}^{-1}\right], C_{p}$ the concentration of peroxide [ $\mathrm{mol}$. $\left.\mathrm{m}^{-3}\right]$, and $I$ was the current [A ].

The current efficiency was the highest at $i=300 \mathrm{~A} \cdot \mathrm{m}^{-2}$, and decreased with increasing current density. Since the increase in the current density made the rate of reaction (2) increase, the current efficiency was considered to decrease by decomposition of hydrogen peroxide.

\subsection{Holdups of gas and liquid phases, and potential profile}

Figure 5 shows the effect of the superficial velocity of gas on the current efficiency and the terminal voltage. At $u_{G}=1.67 \mathrm{~cm} \cdot \mathrm{s}^{-1}$, the current efficiency was the lowest and the terminal voltage was high. Since the highest holdup of the liquid phase increased the thickness of the liquid film on the electrode surface, the reaction resistivity was increased. The current efficiency had a maximum and 

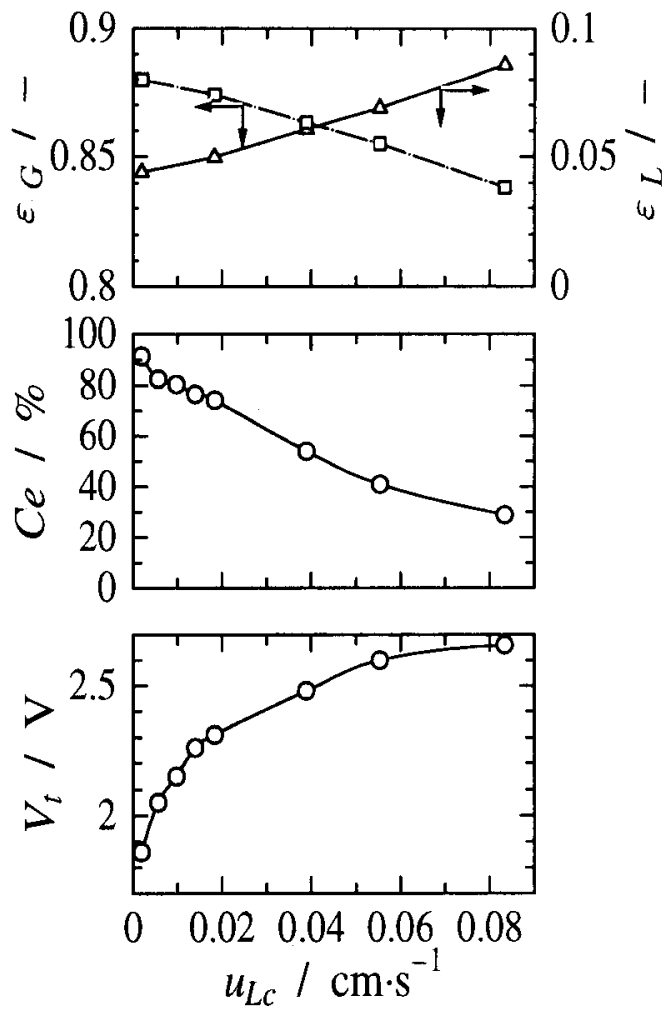

Fig.7 Effects of superficial velocity of catholyte on holdups, current efficiency and terminal voltage. The superficial velocities of anolyte and gas, $u_{L a}$ and $u_{G}$, were 0.03 and $5.0 \mathrm{~cm} \cdot \mathrm{s}^{-1}$ respectively. The current density $i$ was $300 \mathrm{~A} \cdot \mathrm{m}^{-2}$.

the terminal voltage had a mimimun at $u_{G}=$ $5.00 \mathrm{~cm} \cdot \mathrm{s}^{-1}$. Then by increasing $u_{G}$, the current efficiency was decreased because the terminal voltage was increased. Since the effective resistivity of the solution phase was increased by increasing holdups of gas, the potential drop from the separator was supposed to increase. The holdup of the liquid phase was found to be extremely small compared to that of the gas phase. The reason of the composite chip of the hydrophobic carbon used for Dow process ${ }^{10)}$ might be the extremely small liquid holdup around the electrode particle. The higher current density was obtained with the gas-diffusion

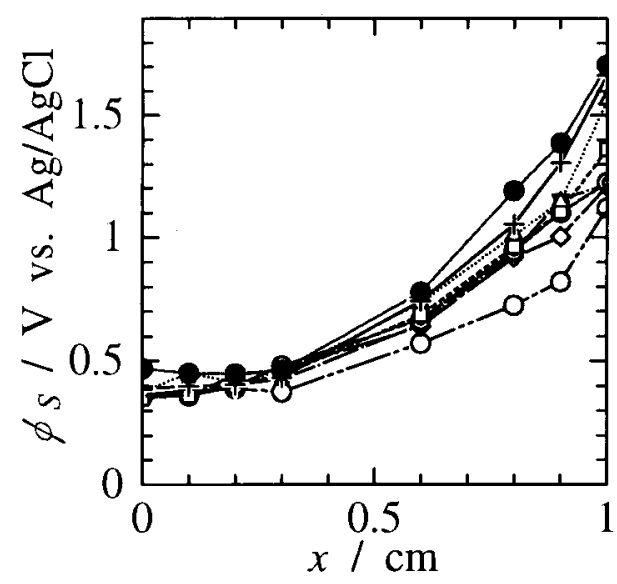

Fig.8 Cathodic potential profiles of solution phase in packed-bed electrode. The superficial velocity of catholyte $u_{L c}$ was $(-0.0833$, (+) $0.0553,(\triangle) 0.0389,(\square) 0.0183,(\bigcirc)$ $0.0140,(\nabla) 0.0097,(\diamond) 0.0057$, and $(\bigcirc)$ $0.0020 \mathrm{~cm} \cdot \mathrm{s}^{-1}$. Experimental condition was the same as in Fig. 7.

electrode $^{6)}$ rather than the packed-bed electrode. The electrolyte penetrating into the inner part of the gas-diffusion electrode was estimated extremely small, and the gas-liquid interface on the intra-electrode surface was supposed to show a significant role to the fast reaction. In the oxygen-sparged felt cathode, the thin liquid film on the intra-electrode surface was considered to be desirable for the fast reaction. Experimental results using different packing materials ${ }^{3)}$ might be influenced by the hydrophobicity of the packing material.

Figure 6 shows the potential profiles of solution phase as a function of the depth in the bed: $x=0$ was feeder side and $x=1$ was separator side. The potential $\phi_{S}$ was lowest at $u_{G}=5.00 \mathrm{~cm} \cdot \mathrm{s}^{-1}$. This increase in $\phi_{S}$ might accelerate the rate of reaction (2) and the current efficiency was decreased as shown in Fig. 5 . The theoretical consideration on the potential profile in the oxygen-sparged cathode will be the subject for a future study.

Figure 7 shows the effect of the superficial velocity of the catholyte $u_{L c}$ on the current 


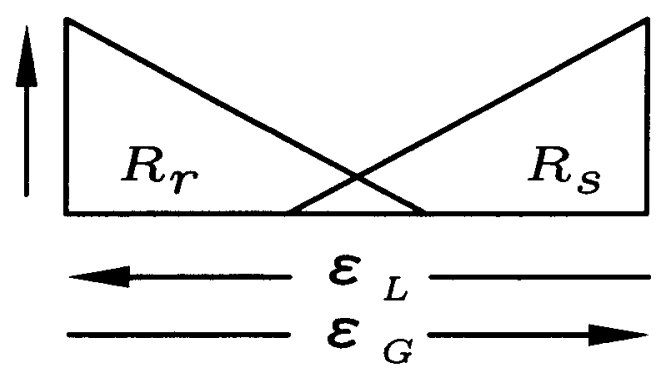

Fig.9 Schematic relationship of resistivities of reaction and solution as a function of holdups of gas and liquid phases; $R_{r}$ : reaction resistivity, $R_{s}$ : effective resistivity of solution.

efficiency and the terminal voltage. The current efficiency was decreased and the terminal voltage was increased by increasing $u_{L c}$. By increasing $u_{L c}$, the holdup of the liquid phase $\varepsilon_{L}$ was increased and the decrease in mass transfer rate of oxygen to the electrode surface resulted in the decrease in generation of hydrogen peroxide. In addition, the increase in the solution resistivity made $\phi_{S}$ shift up, as shown in Fig. 8, and decrease the current efficiency. Since the potential drop of the graphite felt is small due to the high conductivity, the profile of the overpotential in the bed is exclusively dependent to the potential of the solution. Then the high $\phi_{S}$ resulted in the negatively high overpotential, where the peroxide is accelerated to the further reduction by Eq.(2). Figure 9 shows a schematic diagram of reaction and solution resistivities as a function of holdups of gas and liquid phases. The overlapped region for minimum values of both resistivities exists at a certain holdup.

As seen from results of Figs.5 and 7, optimum conditions of holdups of gas and liquid phases were determined as $\varepsilon_{G}=0.85 \sim 0.90$ and $\varepsilon_{L}=0.04 \sim 0.05$. Under these conditions, the production rate of hydrogen peroxide was about $0.9 \mathrm{~g} \cdot \mathrm{h}^{-1}$ at $300 \mathrm{~A} \cdot \mathrm{m}^{-2}$.

\section{CONCLUSION}

The current efficiency was affected by the holdups of gas and liquid phases. The cathodic potential of the solution phase was shifted up by the change in holdups of gas and liquid phases in the packed-bed electrode. The cause of decreasing current efficiency was the reaction of decomposition of hydrogen peroxide, which was accelerated by rise of cathodic overpotential. The optimum conditions for electrolysis were determined as follows: current density was $300 \mathrm{~A} \cdot \mathrm{m}^{-2}$, holdups of gas and liquid phase, $\varepsilon_{G}=0.85 \sim$ 0.90 and $\varepsilon_{L}=0.04 \sim 0.05$.

\section{REFERENCES}

1) C. Oloman, J. Electrochem. Soc., 126 1885 (1979).

2) M. Sudoh, H. Kitaguchi and K. Koide, J. Chem. Eng. Japan, 18, 364 (1985).

3) M. Sudoh, T. Kodera and T. Ichino, J. Chem. Eng. Japan, 24, 165 (1991).

4) M. Sudoh, K. Minamoto, T. Makino and H. Hakamada, J. Chem. Eng. Japan, 24, 465 (1991).

5) M. Sudoh, K. Minamoto, K. Ohashi and K. Noda, J. Chem. Eng. Japan, 25, 170 (1992).

6) H. Takenaka, Y. Kawami, I. Uehara, N. Wakabayashi and M. Motone, Denki Kagaku, 57, 1073 (1989).

7) N. Wakabayashi and H. Takenaka, Denki Kagaku, 61, 1192 (1993).

8) P. C. Foller and R. T. Bombard, J. Appl. Electrochem, 25, 613 (1995).

9) P. Tatapudi and J. M. Fenton, Electrolytic Processes for Pollution Treatment and Pollution Prevention, in Advances in Electrochemical Science and Engineering, (Ed. H. Gerischer and C. W. Tobias), vol.6, VCH Pub, NY, p.363 (1995)

10) G. Brown, D. F. Dong, J. A. McIntyre and R. F. Philips, 1983 Pulp Conference, TAPPI Proceedings, p.341 (1984). 